\title{
Effectiveness of endodontic treatment in patients with generalized periodontitis with prevention of parasympathetic nervous system reactions
}

\author{
Viktor M. Batig ${ }^{1}$, Anatoliy V. Borysenko², Anna I. Prodanchuk', \\ Tatiana A. Hlushchenko ${ }^{1}$, Yuliia H. Kilmukhametova³ ${ }^{3}$ Iryna V. Batih', Olga M. Tokar $^{1}$ \\ ${ }^{1}$ Department of Therapeutic Stomatology, \\ Bukovinian State Medical University, Chernovtsy, Ukraine \\ ${ }^{2}$ Department of Therapeutic Stomatology, \\ O.O. Bohomolets National Medical University, Kyiv, Ukraine \\ ${ }^{3}$ Ukrainian Medical Dental Academy, Poltava, Ukraine
}

\begin{abstract}
Topicality. The prevalence of caries complications (pulpitis and periodontitis) in the population of Ukraine reaches $75-78 \%$. According to date, endodontic treatment is at a high level, but due to some financial difficulties, the population of Ukraine is not receiving it in appropriate level. Improving the quality of endodontic treatment of periodontitis, especially acute and exacerbated ones, is an urgent problem of therapeutic dentistry. Nowadays, the development of treatment methods taking into account the condition of the patient's autonomic nervous system is quite relevant. Purpose. To determine the effectiveness of drug treatment in patients with exacerbated chronic periodontitis and endodontic treatment of teeth with II stage exacerbated course of generalized periodontitis, taking into account the state of the autonomic nervous system, in particular with the predominance of the parasympathetic nervous system. Material and methods. For the clinical study, 30 patients with exacerbated II grade perio-dontitis with periodontal lesions (exacerbated chronic periodontitis) with predominance of the parasympathetic nervous system were selected. Endodontic treatment of 25 teeth with acute course of II grade of generalized periodontitis and 10 teeth with periodontal-periodontal lesions (teeth with signs of exacerbated chronic periodontitis) was conducted in the main group of patients, with a predominance of the parasympathetic section of the autonomic nervous system. The comparison group consisted of 20 patients with predomi-nance of parasympathetic section of autonomic nervous system, which received endodontic treatment of generalized periodontitis (25 teeth) and exacerbated chronic periodontitis (10 teeth), using conventional methods of treatment. All patients were examined using clinical methods.

Results and discussion. The use of the proposed method of endodontic treatment with pre-medication showed a certain decrease in pain in patients after endodontic treatment. X-ray showed complete filling of root canals in all teeth. The statistical analysis showed a greater effectiveness of endodontic treatment in patients in the main group, who under-went premedication.

Conclusions. Conducted clinical study and statistical analysis show the effectiveness of the proposed medicamental premedication during endodontic treatment of teeth in patients with prevalence of parasympathetic section of the autonomic nervous system.
\end{abstract}

Keywords: generalized periodontitis, vegetative nervous system, endodontic treatment

\section{INTRODUCTION}

The prevalence of caries in the population of Ukraine is extremely high and reaches up to $96-98 \%$. This causes a significant number of complications of caries - pulpitis and periodontitis, the occurrence of which is extremely high and reaches $75-78 \%$ [1-5]. The development of acute inflammatory processes in the periodontium causes the further emergence of a number of inflammatory processes of the maxillofacial area. Therefore, the problem of treatment of acute inflammatory processes in the periodontium is relevant for the current state of dental care of the population of Ukraine. To date, endodontic treatment is at a high level, but due to some financial difficulties, the population of Ukraine is not receiving it in a due course. Therefore, the efficacy of endodontic treatment is at a quite low level, due to many complications $[6,8]$. That is why the improvement of the quality of endodontic treatment of periodontitis, especially 
acute and exacerbated, is an urgent problem of therapeutic dentistry in Ukraine [8].

To improve the quality of endodontic treatment of periodontitis, various methods of treatment are used, such as copper-calcium hydroxide depoporosis or cupral [9]. During treating the disease, one should also take into account the individual characteristics of the patient. The emergence and development of the pathological process is significantly influenced by the autonomic nervous system of the person $[10,11]$. According to this, it is relevant to develop methods of treatment of diseases, taking into account the patient's autonomic nervous system, including periodontitis and periodontal diseases.

\section{PURPOSE}

The purpose of this survey is to determine the effectiveness of medicamental treatment in patients with exacerbated chronic periodontitis and endodontic treatment of teeth with II stage exacerbated course of generalized periodontitis, taking into account the state of the autonomic nervous system, in particular with the predominance of the parasympathetic nervous system.

\section{MATERIAL AND METHODS}

For the clinical study, 30 patients with exacerbated II grade periodontitis with periodontal lesions (exacerbated chronic periodontitis) with predominance of the parasympathetic nervous system were selected.

To normalize the state of the autonomic nervous system of patients, a scheme of medical preparation of patients was developed (Utility model patent № 113526), "A method of treatment of patients with acute and exacerbated chronic periodontitis, with the prevalence of the parasympathetic section of the autonomic nervous system" from 25.01.2017.

Patients with predominance of the parasympathetic section of the autonomic nervous system were prescribed appropriate drug treatment two days before treatment and before each visit to the dentist:

- Buskospan $0.01 \mathrm{~g}-1$ tablet thrice a day;

- Valerian tincture - 25 drops three times a day;

- Doxazosin $0.001 \mathrm{~g}-1$ tablet once a day.
After the dental intervention, the course of medication was prescribed for 3-5 days:

- Ibuprofen 0.2 - 2 tablets thrice a day;

- Valerian tincture - 20 drops thrice a day;

- Buskospan $0.01 \mathrm{~g}-1$ tablet three times a day;

- Doxazosin $0.001 \mathrm{~g}-1$ tablet once a day.

In the main group of patients with the predominance of the parasympathetic section of the autonomic nervous system, was conducted endodontic treatment of 25 teeth with acute course of II degree of generalized periodontitis and 10 teeth with periodontal lesion (i.e. teeth with signs of exacerbated chronic periodontitis on the background of exacerbated generalized periodontitis). Among the forms of periodontitis (10 teeth), exacerbated chronic granulating periodontitis was predominant - 9 teeth $(90.0 \%)$, and $1(10.0 \%)$ tooth - exacerbated chronic granulomatous periodontitis. There were 6 $(60.0 \%)$ molars and $4(40.0 \%)$ premolars among them. Among the treated teeth $16(64.00 \%)$ were mandibular incisors, $6(24.0 \%)$ were mandibular premolars, and $3(12.0 \%)$ - mandibular premolars.

All patients were examined using clinical examination methods.

For accurate diagnosis of teeth with periodontal lesions, patients underwent a comprehensive examination of the periodontal condition according to preliminary studies. Contact X - ray was used to diagnose the condition of teeth affected by periodontitis [11]. The $\mathrm{x}$ - ray of the affected teeth made it possible to accu rately assess the condition of the carious cavity, its connection with the tooth cavity, the shape and number of roots and root canals, pathological changes in the periapical tissues. If necessary, thermos-diagnostics and electro-odonto-diagnostics of the affected teeth were performed. The diagnosis of periodontitis was established according to the modern classification [10]. According to the anamnesis, the overall condition of the patients was satisfactory.

Forpreliminaryanalysis of the state of the autonomic nervous system of the patient "Questionnaire to detect vegetative changes" was used [14]. The assessment of the state of the autonomic nervous system was performed by determining the Kerdo index and the vegetative Kerdo index [6].

The comparison group consisted of 20 patients with predominance of parasympathetic section of autonomic nervous system, which received 
endodontic treatment of generalized periodontitis (25 teeth) and exacerbated chronic periodontitis (10 teeth), using conventional methods of treatment.

The proper surgical treatment of exacerbated chronic periodontitis in patients was performed according to the treatment protocols approved by the Ministry of Health of Ukraine (2005). Using an $\mathrm{X}$ - ray the quality of the filling was monitored. The presence and intensity of pain after filling was evaluated in points according to the questionnaire [8].

Patients were examined in the near terms, to evaluate the effectiveness of the treatment. In the case of effective treatment, patients had no complaints, no clinical signs of inflammation in the area of the affected tooth. In the long-term observation on the x-ray, a decrease in the lesion of the periapical tissues was noted.

The intensity of pain after root canal filling was evaluated using the questionnaire developed.

In parallel, patients in both groups with generalized periodontitis underwent the complex treatment using conventional methods, the same as patients in the corresponding main group, but without drug premedication.

\section{RESULTS}

The distribution of teeth by diagnosis and groups of teeth is presented in the table 1 .

Analyzing the conducted questionnaire and clinical examination showed that among patients of the main group with the predominance of the parasympathetic section of the autonomic nervous system (35 teeth), in 4 teeth (11.43\%) was noted pain of low intensity after the endodontic treatment $-1.34+0.41$ points.

In addition, a slight sensitivity during percussion was noted in these teeth. Exacerbation of the process in 2 of these teeth $(5.71 \%)$ was noted during treatment. Similar pain in patients in the comparison group was observed in 8 teeth $(22.86 \%)$. The intensity of pain according to the developed scale in the patients of the comparison group was also within the weak and amounted to $4.67+0.48$ points (table 2 ).

TABLE 2. Intensity of pain in teeth after endodontic treatment in patients with the predominance of the parasympathetic section of the autonomic nervous system $(M \pm m)$

\begin{tabular}{|l|c|c|c|}
\hline \multicolumn{1}{|c|}{ Group } & Number of teeth & Index (points) & $\mathbf{p}_{1}$ \\
\hline Main & 35 & $1.34 \pm 0.41$ & \\
\hline Comparison & 35 & $4.67 \pm 0.48$ & $<0.05$ \\
\hline
\end{tabular}

p1 - is the probability between the data of the main group and the comparison group

Criterion $t=4.839 ; p=0.0001$.

The average duration of pain in patients in the main group with the predominance of the parasympathetic section of the autonomic nervous system during endodontic treatment of periodontal lesions (exacerbated chronic periodontitis) and root canal obturation was 1.5 days. In the case of endodontic treatment of teeth with II degree of generalized periodontitis, the average duration of pain was slightly less and amounted to 1.45 days.

Palpation of the apex area of the treated teeth was painless. X-ray showed complete root canal filling in all teeth. Insignificant overfilled root canals was noted in 3 teeth $(8.57 \%)$ with acute chronic periodontitis in patients of the main group.

In patients of the comparison group with the predominance of the parasympathetic section of the autonomic nervous system, the average duration of pain syndrome after endodontic treatment of periodontal lesions (exacerbated chronic periodontitis) and root canal obturation averaged 2.5 days. In the case of endodontic treatment of

TABLE 1. The distribution of teeth that underwent endodontic treatment in patients with predominance of the parasympathetic section of the autonomic nervous system

\begin{tabular}{|c|c|c|c|c|c|c|c|}
\hline \multirow{2}{*}{$\begin{array}{c}\text { Groups of } \\
\text { patients }\end{array}$} & \multirow{2}{*}{$\begin{array}{c}\text { Number of } \\
\text { teeth }\end{array}$} & \multicolumn{3}{|c|}{ Diagnosis/ Number of teeth } & \multicolumn{3}{c|}{ Group of teeth } \\
\cline { 3 - 8 } & Granulating & Granulomatous & $\begin{array}{c}\text { Generalized } \\
\text { periodontitis }\end{array}$ & Molars & Premolars & Incisors \\
\hline Main & 35 & 9 & 1 & 25 & 6 & 13 & 16 \\
\hline$\%$ & 50.0 & 12.86 & 1.43 & 35.71 & 8.57 & 18.57 & 22.86 \\
\hline Comparison & 35 & 10 & 0 & 25 & 7 & 14 & 14 \\
\hline$\%$ & 50.0 & 14.29 & 0 & 35.71 & 10.0 & 20.0 & 20.0 \\
\hline Overall & 70 & 19 & 1 & 50 & 13 & 27 & 30 \\
\hline$\%$ & 100.00 & 27.15 & 1.43 & 71.42 & 18.57 & 38.57 & 42.86 \\
\hline
\end{tabular}


teeth with II grade of generalized periodontitis, the average duration of pain was slightly shorter, and was 2.7 days. The duration of pain in patients in the comparison group was 2.7 days, in the case of endodontic treatment of generalized periodontitis, it was 2.9 days. X-ray showed that the root canals of the teeth are filled up to the apical narrowing. Insignificant excretion of filling material over the apex was noted in $3(8.57 \%)$ teeth in patients of the comparison group.

A comparison of the relative number of complications (pain) was made, to determine the effectiveness and superiority of the proposed treatments using the $\chi 2$ criterion (xi-square). It was noted in $4(11.43 \%)$ of 35 teeth in patients in the main group, and in $8(22.86 \%)$ of 35 teeth in patients of comparison group with the predominance of the parasympathetic section of autonomic nervous system. The number of closest complications in patients of the main group was 4 out of 35 , and in patients of comparison group -8 out of 35 . Thus, the frequency of complications in both groups was $(4+8) / 70 \times 100=17.14 \%$. The expected calculated number of immediate complications in patients of the main group was: 17.14 / $31 \times 100=55.29 \%$.

The expected calculated number of immediate complications in patients in the comparison group was: $17.14 / 27 \times 100=63.48 \%$.

The obtained results revealed a higher expected number of closest complications in the comparison group patients than in the main group patients.

\section{DISCUSSION}

Periodontitis is a common, chronic, multifactorial, inflammatory condition affecting the supporting tissues of the teeth. It leads to the progressive destruction of the tooth supporting apparatus and eventual tooth loss. There is now overwhelming evidence that periodontitis has a negative impact on oral health-related quality of life [1-7]. Significant impacts have been shown in the functional, physical, social and psychological domains. Severe periodontitis appears to have a greater impact than mild to moderate periodontitis [8-12].

It is important to be aware of this impact when assessing patients in practice. Discussions might include psychological concerns, halitosis, pain, and poor aesthetics [13-19]. The periodontal treatment plan should be based on both the needs diagnosed by the clinician but also those perceived by the patient [6-9].

The latest global burden of diseases, injuries and risk factors' study demonstrated that, from 354 diseases/injuries measured across 195 countries, the greatest age-standardised prevalence of years lived with disease' in both 1990 and 2017 were oral disorders, headache disorders, and tuberculosis including latent tuberculosis [13-15]. The periodontal data were true for both males and females and, unlike caries where age-standardised percentage rates had decreased, in periodontal diseases rates had increased between 2008 and 2018 [1-4,16].

The clinical difference between chronic and aggressive periodontitis especially in generalized forms is not clear $[17,18]$. The extent and amount of clinical inflammation in generalized aggressive periodontitis appear to be similar to that observed in chronic periodontitis [19]. At that point, clear diagnostic distinction can be made according to age of onset and patients' family history [20].

Recently, continuous multilevel risk assessmentbased prognostication systems were proposed $[20,21]$. In the light of these evidences, it seems possible to retain a tooth with negative prognosis in a well-maintained cooperative patient [22-24]. In this research, all teeth were treated periodontally and retained with multidisciplinary perio-endo treatment, and favorable clinical improvements were obtained to change their prognosis at 1 -year from "hopeless" to "maintainable" condition. Only one premolar tooth was extracted at the end of the 7 th year due to the increasing mobility and tooth number 36 revealed periapical radiolucency on the radiograph without any clinical symptoms.

Based on existing evidence, the American Academy of Periodontology suggested several indications for gingival augmentation procedures [25]. We placed FGG onto the insufficient keratinized tissue zones after IPT before flap surgeries.

Today, by the help of various new technologies, biological approaches and biomaterials, the challenge is now to introduce the experience and knowledge contributing to patient outcomes in terms of function, ease of care, esthetics, and longterm maintenance [26]. There are various biomaterial combinations used in the regeneration of periodontal bone defects [27-31]. It has been shown 
that the use of EMD combined with bone graft materials is promising and useful for periodontal regeneration [27]. In this research, EMD + BDX was used as the regenerative combination. The results of a recent study showed that hopeless teeth can be treated successfully with various regenerative combinations and maintained over a period of 5 years in health and function [31]. The present case report parallel with the aforementioned study revealed encouraging results for regenerative interventions to be considered as suitable alternatives to the extraction of severely compromised teeth with intrabody defects to or beyond the root apex [31]. However, the importance of patient selection, clinicians' experience and skill, the scientific evidence, treatment plan, a cost/benefit analysis and a strict periodontal supportive care program must be underlined as the keys for the success of

\section{REFERENCES}

1. Chapple ILC, Mealey BL, Van Dyke TE et al. Periodontal health and gingival diseases and conditions on an intact and a reduced periodontium: Consensus report of workgroup 1 of the 2017 World Workshop on the Classification of Periodontal and Peri-Implant Diseases and Conditions. J Clin Periodontol 2018; 45 Suppl 20: 68-77.

2. Araujo A C, Gusmão E S, Batista J E, Cimões R. Impact of periodontal disease on quality of life. Quintessence Int 2010; 41:111-118.

3. Papapanou PN, Sanz M, Buduneli N et al. Periodontitis: Consensus report of workgroup 2 of the 2017 World Workshop on the Classification of Periodontal and Peri-Implant Diseases and Conditions. J Clin Periodontol 2018; 45 Suppl 20:162-170.

4. Bernabe $\mathrm{E}$, Marcenes W. Periodontal disease and quality of life in British adults. J Clin Periodontol 2010; 37:968-972.

5. Matuliene G, Pjetursson BE, Salvi GE et al. Influence of residual pockets on progression of periodontitis and tooth loss: Results after 11 years of maintenance. J Clin Periodontol 2008; 35:685-695.

6. Martsenyuk VP, Vakulenko DV, Vakulenko LO. Kerdo informative index to determine the level of violations of autonomic regulation at osteochondrosis of the cervical spine. Medical Informatics and Engineering 2013, (1). https://doi.org/10.11603/mie.19961960.2013.1.418.

7. Jepsen S, Caton JG, Albandar JM et al. Periodontal manifestations of systemic diseases and developmental and acquired conditions: Consensus report of workgroup 3 of the 2017 World Workshop on the Classification of Periodontal and Peri-Implant Diseases and Conditions. J Clin Periodontol 2018; 45 Suppl 20:219-229.

8. Martyniuk $O$, Vilyanskiy V. Assessment of students' health condition by indicators of adaptation potential, biological age and bio-energetic reserves of organism. Physical education of students. 2015; 3: 20-29. URL: http://nbuv.gov.ua/UJRN/PhVSTSE_2015_3_5.

9. Machtei EE, Hausmann E, Dunford R et al. Longitudinal study of predictive factors for periodontal disease and tooth loss. $J$ Clin Periodontol 1999; 26:374-380.

10. Needleman I, McGrath C, Floyd P, Biddle A. Impact of oral health on the life quality of periodontal patients. J Clin Periodontol 2004; $31: 454-457$. periodontal treatment approaches [31]. Although periodontists are recognized as more skilled in predicting tooth prognosis, we must remember that treatment protocol does not work in any case if the patient compliance does not exist [19].

\section{CONCLUSIONS}

The clinical study and the statistical analysis show that the differences in the frequency of closest complications in the main group and the comparison group are not accidental. The reliability of the findings exceeds $95 \%$. These results confirm the effectiveness of the proposed version of premedication during endodontic treatment of teeth in patients of the main group with the predominance of the parasympathetic section of the autonomic nervous system.

Conflict of interest: none declared Financial support: none declared

11. White DA, Tsakos G, Pitts NB et al. Adult Dental Health Survey 2009: Common oral health conditions and their impact on the population. Br Dent J 2012; 213:567-572.

12. Nociti FH Jr, Casati MZ, Duarte PM. Current perspective of the impact of smoking on the progression and treatment of periodontitis. Periodontol 2000 2015; 67:187-210.

13. Dietrich $T$, Ower $P$, Tank $M$ et al. Periodontal diagnosis in the context of the 2017 classification system of periodontal diseases and conditions - implementation in clinical practice. Br Dent J 2019; 226:16-26.

14. Newton J T, Asimakopoulou K. The perceived acceptability of the DEPPA patient assessment tool: A questionnaire survey of Denplan Excel patients. Br Dent J 2017; 222:767-770.

15. Suvan J E. Effectiveness of mechanical nonsurgical pocket therapy. Periodontol 2000 2005; 37:48-71.

16. Sagar A. Full mouth versus quadrant treatment in chronic periodontitis. Prim Dent J 2014; 3: 66-69.

17. Lang NP, Tan WC, Krähenmann MA, Zwahlen M. A systematic review of the effects of full-mouth debridement with and without antiseptics in patients with chronic periodontitis. J Clin Periodontol 2008; 35 (Spec Iss):8-21.

18. Kornman KS. Mapping the pathogenesis of periodontitis: A new look. J Periodontol. 2008;79(8 Suppl):1560-8.

19. Matuliene G, Pjetursson BE, Salvi GE, Schmidlin K, Brägger U, Zwahlen $\mathrm{M}$ et al. Influence of residual pockets on progression of periodontitis and tooth loss: Results after 11 years of maintenance. J Clin Periodontol. 2008;35:685-95.

20. Kao RT. Strategic extraction: A paradigm shift that is changing our profession. J Periodontol. 2008;79:971-7.

21. Kwok V, Caton JG. Commentary: Prognosis revisited -A system for assigning periodontal prognosis. J Periodontol. 2007;78:2063-71.

22. Armitage GC, Cullinan MP. Comparison of the clinical features of chronic and aggressive periodontitis. Periodontol 2000. 2010;53:12-27.

23. Lang NP, Tonetti MS. Periodontal diagnosis in treated periodontitis. Why, when and how to use clinical parameters. J Clin Periodontol. 1996;23:240-50.

24. DeVore CH, Beck FM, Horton JE. Retained "hopeless" teeth. Effects on the proximal periodontium of adjacent teeth. $J$ Periodontol. 1988;59:647-51. 
25. Consensus report. Mucogingival therapy. Ann Periodontol. 1996; 1:702-6.

26. Yilmaz S, Kuru B, Altuna-Kiraç E. Enamel matrix proteins in the treatment of periodontal sites with horizontal type of bone loss. $J$ Clin Periodontol. 2003;30:197-206

27. Meseli SE, Agrali OB, Peker O, Kuru L. Treatment of lateral periodontal cyst with guided tissue regeneration. Eur J Dent. 2014; 8:419-23.

28. Sumer M, Keles GC, Cetinkaya BO, Balli U, Pamuk F, Uckan S. Autogenous cortical bone and bioactive glass grafting for treatment of intraosseous periodontal defects. Eur J Dent. 2013;7:6-14.

29. Keles GC, Sumer M, Cetinkaya BO, Tutkun F, Simsek SB. Effect of autogenous cortical bone grafting in conjunction with guided tissue regeneration in the treatment of intraosseous periodontal defects. Eur J Dent. 2010;4:403-11.

30. Li W, Xiao L, Hu J. The use of enamel matrix derivative alone versus in combination with bone grafts to treat patients with periodontal intrabony defects: A meta-analysis. J Am Dent Assoc. 2012; 143:e46-56.

31. Cortellini P, Stalpers G, Mollo A, Tonetti MS. Periodontal regeneration versus extraction and prosthetic replacement of teeth severely compromised by attachment loss to the apex: 5-year results of an ongoing randomized clinical trial. J Clin Periodontol. 2011;38:915-24. 